\title{
A Robust Vibration Control of a Magnetorheological Damper Based Railway Suspension Using a Novel Adaptive Type 2 Fuzzy Sliding Mode Controller
}

\author{
Sy Dung Nguyen, ${ }^{1,2}$ Dongsoo Jung, ${ }^{3}$ and Seung-Bok Choi ${ }^{3}$ \\ ${ }^{1}$ Division of Computational Mechatronics, Institute for Computational Science, Ton Duc Thang University, Ho Chi Minh City, Vietnam \\ ${ }^{2}$ Faculty of Electrical and Electronics Engineering, Ton Duc Thang University, Ho Chi Minh City, Vietnam \\ ${ }^{3}$ Department of Mechanical Engineering, Inha University, Incheon, Republic of Korea \\ Correspondence should be addressed to Seung-Bok Choi; seungbok@inha.ac.kr
}

Received 8 March 2017; Revised 31 July 2017; Accepted 13 August 2017; Published 17 October 2017

Academic Editor: Athanasios Chasalevris

Copyright (C) 2017 Sy Dung Nguyen et al. This is an open access article distributed under the Creative Commons Attribution License, which permits unrestricted use, distribution, and reproduction in any medium, provided the original work is properly cited.

\begin{abstract}
This work proposes a novel adaptive type 2 fuzzy sliding controller (AT2FC) for vibration control of magnetorheological damper(MRD-) based railway suspensions subjected to uncertainty and disturbance (UAD). The AT2FC is constituted of four main parts. The first one is a sliding mode controller (SMC) for specifying the main damping force supporting the suspension. This controller is designed via Lyapunov stability theory. The second one is an interpolation model based on an interval type 2 fuzzy logic system for determination of optimal parameters of the SMC. The third one is a nonlinear UAD observer to compensate for external disturbances. The fourth one is an inverse MRD model (T2F-I-MRD) for specifying the input current. In the operating process, an adaptively optimal structure deriving from the SMC is created (called the Ad-op-SMC) to adapt to the real status. Working as an actuator, the input current for MRD is then determined by the T2F-I-MRD to generate the required damping force which is estimated by the Ad-op-SMC and the nonlinear observer. It is shown that the obtained survey results reflect the AT2FC's excellent vibration control performance compared with the other controllers.
\end{abstract}

\section{Introduction}

Controlling effectively nonlinear systems subjected to UAD such as MRD-based railway suspensions is always challenging issue to be effectively resolved. Being seen as noise, UAD may come from several reasons, model uncertainties, lack of accuracy of the measurement devices, unknown nonlinear characteristics of the actuators, and unknown impact of the operating environment. They are all becoming a big issue to achieve accurate and high control performance. Operating in this condition, the design of an appropriate adaptive controller to compensate uncertainties and noises is absolutely required to guarantee robust stability. Focusing on this, various studies have been carried out. For example, applying the sliding mode technique [1-5], interpolating control rules of fuzzy logic [6-13], or building observers to compensate for the influence of UAD has obtained positive results [14-17].
Besides, combining these methods has been also seen as the appropriate approach providing some advantages [18-22].

As well known, the sliding mode control (SMC) method could surmount difficulties related to the nonlinear and uncertainty aspects. With significant potentials, SMC controllers have been widely used for nonlinear control systems $[1-5,9,12]$. Using a proper control strategy, a process consisting of two phases, the approaching and maintaining, is performed in order to set up and uphold reasonable operating conditions. In the first one, the system is controlled to direct towards the sliding surface, and then the system dynamics switches along it in the second phase. To perform this, as usual, an infinite commutation function is required to create the control signal. This sometimes causes the chattering phenomenon impacting negatively on the system $[9,18]$. To overcome this, fuzzy logic systems (FLSs) have been used as a reasonable tool for establishing solutions [18-22]. Therefore 
there have been several controllers built by combining the SMC and FLS, in which FLS is used to establish control laws and/or to build the black-box-typed models depicting the system's input-output relation $[8,9,22]$. Regarding these applications, both the type 1 fuzzy logic system (T-1FLS) and the type 2 fuzzy logic system (T-2FLS) can all be utilized. However, reality shows that, in case of having to cope with difficulties related to UAD or noise, T-2FLS has significant advantages compared with the other [7, 12, 13, 23-27].

The T-2FLS is built based on type 2 fuzzy sets (T-2FSs). In general, a fuzzy number of $[0,1]$ is used in the T-2FS. In fact, if an element cannot determine its membership in a set as 0 or 1 , T1-FS can be used. However, if the membership grade of an element in a set even cannot be expressed as a crisp number in $[0,1]$, the T-2FS can be seen as a useful option [7]. By using the T-2FS, uncertainty aspects can be depicted by the shape or the parameters of the fuzzy sets or both of them [23]. It is noted that if fuzzy rules are formed from noisy data set or built via an uncertainty relation of a chosen physical model being operated in a disturbed environment, the created rules will be carried over by these statuses. As a result, this causes decreasing of the effectiveness of the established FLS. In this case, IT-2FLSs based T-2FSs can depict the system better than T-1FLSs [7]. These aspects should be considered to build controllers for railway suspension systems, where UAD always exists and influences negatively on the controllers $[9,27]$. In [9], in order to cope with the unknown load time varying and the model error, a controller named NFSmUoC for MRD-based railway suspensions was built using an adaptive neuro-fuzzy inference system (ANFIS), sliding mode control, and an uncertainty observer, in which the ANFIS worked as a T-1FLS to identify the MRD. By analyzing the results obtained from this work we observe that the optimal values of the sloping rate as well as the changing rate of the sliding surface are quite sensitive to the special features of the real track type and the load. Besides, there exists a mutual dependence between dynamic response of the MRD and the environment condition including temperature, to which the ANFIS working as a T-1FLS cannot be able to express fully this multidirectional and nonlinear relation. Hence, in this case, building the control ability to adapt to UAD based on SMC and IT-2FLS can be seen as an appropriate option. Paying attention to this aspect, another approach which relied on building the capability to predict the track profile's status to update adaptively the optimal parameters of the control system was presented in [2], by which the unwanted impacting of UAD can be overcome partly. Its effectiveness, however, is reduced if the train speed increases. The main reason is the relative delay between the real and predicted track status.

Consequently, in this paper a new adaptive type 2 fuzzy sliding controller (AT2FC) for controlling vibration of MRD railway suspension systems subjected to UAD is presented. UAD consists of the unknown load change including the random impact of wind force, unknown aspects of the track profiles, and the model error. The main technical contributions of this work are summarized as follows. The first one is an adaptively optimal SMC named Ad-op-SMC for specifying the main damping force supporting the suspension. First, the initial structure of SMC is designed based on Lyapunov stability theory, and then, in order to improve the adaptive ability to cope with UAD, we propose an interpolation model called T2F-SMC-TPM which relied on an IT-2FLS for interpolating the optimal parameters of the SMC according to the real status of the track profile and unsprung mass. The second distribution is an IT-2FLS-based inverse MRD model (T2F-I-MRD) established via the measured dynamic response of a real MRD for specifying the input current supporting the MRD to generate the required damping force. During the operating process, the optimal parameters of the Ad-op-SMC are always updated by the T2F-SMC-TPM to adapt itself to the real status of the track and unsprung mass. By using the required damping force value specified by the Ad-op-SMC and NUO in total, the corresponding input current for the MRD is then estimated by the T2F-IMRD to stamp out the chassis mass's vibration. It should be noted that, together with the main roles in the T2F-SMCTPM and T2F-I-MRD as mentioned above, the IT-2FLSs are also taking part in reducing the system's model error via the ability to infer of the type 2 fuzzy logic system.

\section{Building an IT-2FLS from a Data Set}

In this section, the algorithm for optimal design of IT-2FLS from a data set named OD-T2FLS [7] is briefly presented to build the T2F-SMC-TPM and T2F-I-MRD in the next section. The accuracy of a data-driven fuzzy model depends directly on the accuracy rate of the fuzzy sets used to establish the FLS. Since it is very difficult to exactly determine an appropriate membership grade of an element due to many causes related to understanding grade of the system or uncertainty issues, T-2FS can be generally used. $\widetilde{A}$ in the T-2FS is defined as follows [7]:

$$
\begin{aligned}
\widetilde{A} & =\int_{x \in X} \int_{u \in J_{x}} \frac{\mu_{\widetilde{A}}(x, u)}{(x, u)} \\
& =\left\{\left((x, u), \mu_{\widetilde{A}}(x, u)\right) \mid \forall x \in X, \forall u \in J_{x} \subseteq[0,1]\right\},
\end{aligned}
$$

where $0 \leq \mu_{\widetilde{A}}(x, u) \leq 1, x \in X$ and $u \in J_{x} \subseteq[0,1]$, is a type 2 membership function (T-2MF). When $\mu_{\widetilde{A}}(x, u)=1 \forall(x, u)$, $\widetilde{A}$ is called an interval T-2FS (IT-2FS) and given by

$$
\widetilde{A}=\int_{x \in X} \int_{u \in J_{x}} \frac{1}{(x, u)}: J_{x} \subseteq[0,1] .
$$

The secondary membership function (MF) of a T-2FS $\widetilde{A}$ is a vertical slice of $\mu_{\widetilde{A}}(x, u)$. Figure 1 reflects that, at each value of $x$, for example, $x=x^{\prime}$, the 2D plane, whose axes are $u$ and $\mu_{\widetilde{A}}\left(x^{\prime}, u\right)$, is called a vertical slice of $\mu_{\widetilde{A}}(x, u)$ at $x^{\prime}$. The primary membership of $x, J_{x}$, is called the domain of the secondary MF.

The footprint of uncertainty (FOU) is used to express the uncertainty status in the primary memberships. This is a bounded region, which is the union of all primary memberships:

$$
\operatorname{FOU}(\widetilde{A})=\bigcup_{x \in X} J_{x}
$$




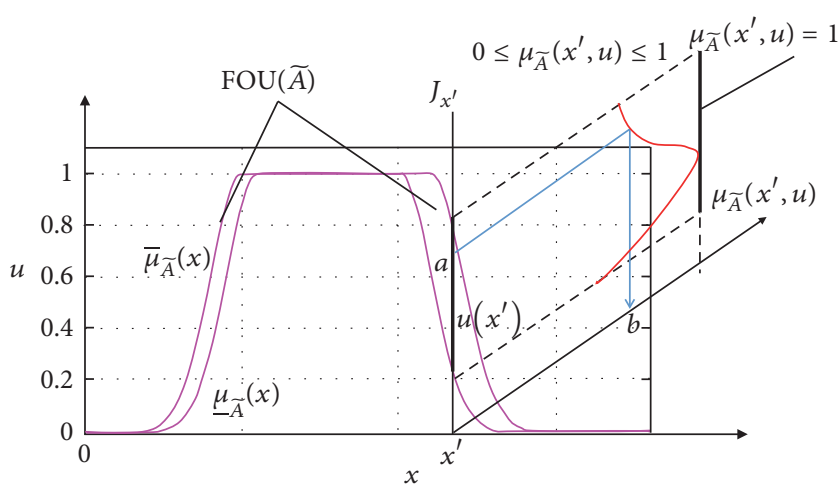

FIGURE 1: Expressing an IT-2FS and some relative aspects.

$\mathrm{FOU}(\widetilde{A})$ in (3) is the region bounded by two primary membership functions, upper one (UMF) and lower one (LMF), as follows:

$$
\begin{aligned}
& \bar{\mu}_{\widetilde{A}}(x) \equiv \overline{\operatorname{FOU}}(\widetilde{A}), \\
& \underline{\mu}_{\widetilde{A}}(x)=\underline{\operatorname{FOU}}(\widetilde{A}) .
\end{aligned}
$$

IT-2FLS is a FLS based on the IT-2FSs. In the IT-2FLS, the significant factors to be considered are falsifiers, inference engine, rules, output producer including a type reducer, and a defuzzifier. These can be expressed by a mapping from crisp inputs into T-2FSs, and from T-2FSs into crisp outputs. The mapping can be expressed via the general form of IF-THEN rules. The $k$ th rule of $\bar{x}_{i}$ can be displayed as follows:

$$
R^{(k)}: x_{i 1} \text { is } \widetilde{B}_{1}^{(k)} \text { and } \ldots \text { and } x_{i n} \text { is } \widetilde{B}_{n}^{(k)} \text {, then } y \text { is } \widetilde{G}^{(k)} \text {, }
$$

where $k=1 \cdots M$, and $M$ denotes the number of rules, $\bar{x}_{i}=$ $\left[x_{i 1}, \ldots, x_{i n}\right]$ is the input of the $i$ th data sample, $\widetilde{B}_{1}, \ldots, \widetilde{B}_{n}$ are antecedent T-2FSs, and $\widetilde{G}^{(k)}$ is a consequent T-2FS corresponding to the $k$ th rule. The predicted output is calculated via an output producer consisting of type reducer and defuzzifier. This is a process of mapping from the T-2FS to the T-1FS and hence to the crisp output of the T-2FLS. There are various approaches for defining the crisp output, in which the centroid method is more widely applied and also used in this study. As a result, a crisp number expressing the center of the type-reduced set is the output of the defuzzifier which indicates the predicted output.

In [7], Nguyen et al. presented algorithm OD-T2FLS for optimal design of the IT-2FLS from a dataset. This is a combination of the clustering in joint input-output data space to establish and optimize the T-1FLS and an adaptive solution to transforming the T-1FLS into the IT-2FLS including the optimizing parameters of the IT-2FLS. Firstly, a process of establishing ANFIS is performed. The ANFIS being T-1FLS is then optimized via the training process. The parameters of the optimized T-1FLS are then used to build the initial structure of the IT-2FLS. Subsequently, the IT-2FLS is optimized via the well-known genetic algorithm (GA). The content of the OD-T2FLS can be briefly presented by the flowchart shown in Figure 2.

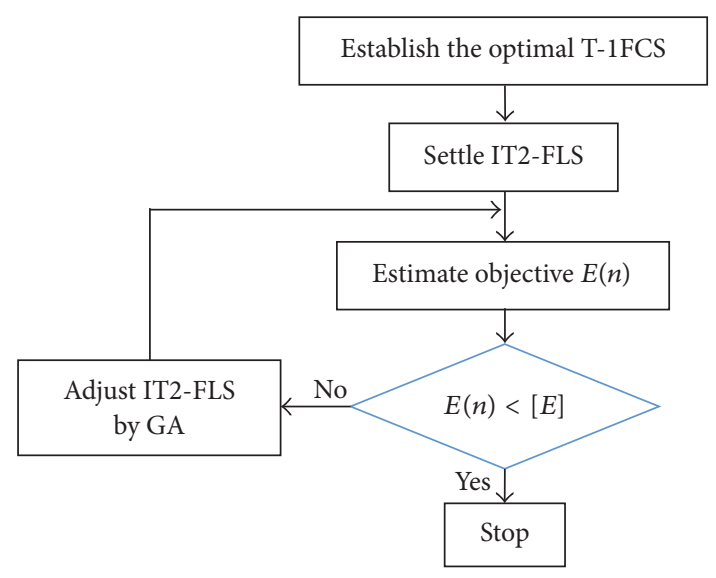

Figure 2: The flowchart of the algorithm OD-T2FLS.

\section{Vibration Control Problem and Solution}

In this work, MRD-based railway suspension system which can be modelled as a quarter rail car is adopted to demonstrate robust vibration control performance of the proposed control approach. This is a nonlinear system impacted by UAD which can be participated into two groups. The first one relates to the unknown model error as well as time load including weight of passengers and impacting of wind on the operating train. The second group relates to unknown uneven status of track profiles. The impacting of the first group on the system is illustrated by the parameter $d=d(t)$ in the figure while that of the second group is expressed by the vertical displacement of track profile, $z_{r}$. Displacements of $m_{s}$ and $m_{u}$ are presented by $z_{s}$ and $z_{u}$, respectively. In this model, $u$ is the active control force generated by the actuator which is the MRD in this survey; $k_{s}$ is the stiffness coefficient of linear spring; $k_{t}$ is deflection stiffness the wheel and track; $c_{s}$ is the damping coefficient of suspension; $c_{t}$ is the damping coefficient of the wheel and track. $f_{s}, f_{t s}, f_{d}$, and $f_{t d}$ are sprung and damper forces corresponding to $k_{s}, k_{t}, c_{s}$, and $c_{t}$, respectively. The unsprung mass $m_{u}$ consisting of mass of the wheel, shaft, brake, and suspension linkage is constant while the sprung mass $m_{s}$ is the time parameter, $m_{s}=m_{s}(t)$.

A state vector $\mathbf{x}(t)$ having state verifies depicted via dynamic response signals of $m_{s}(t)$ and $m_{u}$ is defined as follows:

$$
\begin{array}{r}
\mathbf{x}(t)=\left[x_{1}, x_{2}, x_{3}, x_{4}\right]^{T} \\
x_{1}=z_{s}, x_{2}=\dot{z}_{s}, x_{3}=z_{u}, x_{4}=\dot{z}_{u} .
\end{array}
$$

Based on $\mathbf{x}(t)$, the spring and damper forces can be expressed as

$$
\begin{aligned}
& f_{s}=k_{s}\left(x_{1}-x_{3}\right) \\
& f_{d}=c_{s}\left(x_{2}-x_{4}\right) \\
& f_{r}=f_{t s}+f_{t d} \\
& f_{t s}=k_{t}\left(x_{3}-z_{r}\right) \\
& f_{t d}=c_{t}\left(x_{4}-\dot{z}_{r}\right) .
\end{aligned}
$$


The dynamic equations of the sprung and unsprung masses can be established via the Newton's Second Law as below [2]:

$$
\begin{aligned}
m_{s}(t) \ddot{z}_{s}+f_{s}+f_{d} & =-u+d \\
m_{u} \ddot{z}_{u}-f_{s}-f_{d}+f_{r} & =u .
\end{aligned}
$$

By using expressions from (6) to (9), the state space can be reexpressed by (10) and (11) as below:

$$
\begin{aligned}
& \dot{\mathbf{x}}(t)=\mathbf{f}(\mathbf{x}(t))+\mathbf{g}_{1}(\mathbf{x}(t)) u(t)+\mathbf{g}_{2}(\mathbf{x}(t)) d(t) \\
& y(t)=h(\mathbf{x}(t)),
\end{aligned}
$$

where

$$
\begin{aligned}
& \mathbf{f}(x)=\left[\begin{array}{c}
x_{2}(t) \\
-\frac{F_{s}}{m_{s}(t)} \\
x_{4}(t) \\
\frac{F_{s}-F_{r}}{m_{u}}
\end{array}\right], \\
& \mathbf{g}_{1}=\left[\begin{array}{c}
0 \\
-\frac{1}{m_{s}(t)} \\
0 \\
\frac{1}{m_{u}}
\end{array}\right], \\
& \mathbf{g}_{2}=\left[\begin{array}{c}
0 \\
\frac{1}{m_{s}(t)} \\
0 \\
0
\end{array}\right], \\
& h(\mathbf{x}(t))=x_{1}(t), \\
& F_{r}=f_{t s}+f_{t d} ; F_{s}=f_{s}+f_{d} .
\end{aligned}
$$

The role of the controller AT2FC is to create the control force $u=u(t)$ such that the acceleration of vertical dynamics vibration response of the car chassis can be extinguished as much as possible. Focusing on this purpose, in this paper a robust control strategy for the MRD which works as an actuator to create the control force $u=u(t)$ is proposed as shown in Figure 4.

\section{Design of the Controller AT2FC}

The basic structure and operating principle of the proposed controller AT2FC is shown in Figure 5. The control algorithm consists of four main parts: the sliding mode controller SMC, the identification model T2F-SMC-TPM, the nonlinear UAD observer NUO, and the inverse MRD model T2F-I-MRD. At each time, related to the real-time-based status of the load and track profile, a corresponding adaptively optimal structure of the SMC, the Ad-op-SMC, is established via the T2F-SMCTPM to specify the main control force $u_{s}(t)$. The impact of the disturbance aspects on the suspension is compensated by the compensating active force $u_{c}(t)$ estimated by the NUO. Working as an actuator, the input current $I(t)$ for MRD is then estimated via the T2F-I-MRD based on the required total input force value coming from the Ad-op-SMC and NUO; $u(t)=u_{s}(t)+u_{c}(t)$.

4.1. Design of the Original SMC. For the design of original SMC, as presented in [2], a sliding surface based on displacement and velocity of the chassis is firstly defined as follows:

$$
S=k_{1} x_{1}+x_{2}
$$

where $k_{1}$ is a positive parameter participating in $S$ as a rotation coefficient of the sliding surface. By choosing Lyapunov candidate function as $V(\mathbf{x})=0.5 S^{2} \geq 0$, to which $\dot{V}(\mathbf{x})=S \dot{S}$, it can infer that control law $u(t)$ is chosen so that $\dot{S}(t)=-\rho \operatorname{sat}(S(t))$, in which $\rho$ is a positive real number; then $V(\mathbf{x}) \rightarrow 0$ is a Lyapunov asymptotically stable process. With reference to (8), it can infer the control law deriving from the original SMC as follows:

$$
\begin{aligned}
u(t)= & k_{1} m_{s}(t) x_{2}(t)-f_{s}-f_{d}+\rho m_{s}(t) \operatorname{sat}(S(t)) \\
& +\widehat{d}(t)
\end{aligned}
$$

where $\widehat{d}(t)$ denotes the estimated value of $d(t)$; the function sat $(\cdot)$ is defined as

$$
\text { sat }(S)= \begin{cases}1 & \text { if } S>1 \\ S & \text { if }-1 \leq S \leq 1 \\ -1 & \text { if } S<-1 .\end{cases}
$$

Lemma 1. Consider the system (10) controlled by active force (13). The dynamics of $S(t) \rightarrow 0$ is an asymptotically stable process in the sense of Lyapunov process if and only if $\widehat{d}(t) \rightarrow$ $d(t)$.

Proof. $\widehat{d}(t)$ can be expressed via the estimate error $e(t) \in \mathfrak{R}$ of $d(t)$ as below:

$$
\widehat{d}(t)=d(t)-e(t)
$$

From (8), (12), (13), and (15), the following equations are inferred:

$$
\dot{S}(t)=-\rho \operatorname{sat}(S(t))+\frac{e(t)}{m_{s}(t)} .
$$

By choosing a Lyapunov candidate function as $V(S(t))=$ $0.5[S(t)]^{2} \geq 0$ together with (16), the following is obtained:

$$
\dot{V}(S(t))=-\rho|S(t)|+\frac{S(t) e(t)}{m_{s}(t)} .
$$

Based on (17), if $e(t)=0$, meaning $\widehat{d}(t) \rightarrow d(t)$, we obtain

$$
\dot{V}(S(t))=-\rho|S(t)| \leq 0 .
$$


Due to $V(S(t)) \geq 0$, it can infer that $S(t) \rightarrow 0$ is an asymptotically stable Lyapunov process. Conversely, if $e(t) \neq$ 0 is a random real number, the clause (18) does not exist. This means that $S(t) \rightarrow 0$ is not an asymptotically stable Lyapunov process. Practically, in order to overcome difficulty related to the chattering phenomenon, we use $\rho$ in (18) as proposed by S. D. Nguyen and Q. H. Nguyen in [9] as in (19).

$$
\rho=k_{2}\left(1-\exp \left(-k_{3}\left|x_{2}\right|\right)\right) \geq 0 \quad \forall x_{2},
$$

where $k_{i}, i=2,3$, are positive adaptive coefficients. In this paper, $k_{3}$ is chosen by 0.3 . The optimal value of $\rho$ such that $S(t) \rightarrow 0$ can be determined via the well-known optimal seeking algorithms. In this paper, we use the rank-Differential Evolution algorithm [28] for this work to build the T2F-SMCTPM which will be presented in the next section by estimating $\widehat{d}$ so that Lemma 1 is satisfied.

4.2. Design of the NUO. The role of the NUO is to estimate $d=d(t)$, meaning $\widehat{d}$ in (13). In [9], based on the theory of nonlinear dynamic inversion control presented in [17], S. D. Nguyen and Q. H. Nguyen have presented the NUO for estimating the impact of lumped disturbance aspects. By this way, the compensating active force is expressed by

$$
\widehat{d}(t)=z(x, t)+p(x)=z(x, t)+\mathbf{1}^{T} \mathbf{x}(t),
$$

where $\mathbf{1}=\left[\begin{array}{llll}l_{1} & l_{2} & l_{3} & l_{4}\end{array}\right]^{T}$ is a vector of constants and $z(x, t)$ can be estimated via a recurrent process based on the following expression:

$$
\begin{aligned}
\dot{z}(x, t)= & \left(\frac{l_{2}}{m_{s}(t)}-\frac{l_{4}}{m_{u}}\right)(z+p+u)-l_{1} x_{2} \\
& +\frac{l_{2}}{m_{s}(t)}\left(f_{s}+f_{d}\right)-l_{3} x_{4} \\
& -\frac{l_{4}}{m_{u}}\left(f_{s}+f_{d}-f_{r}\right) .
\end{aligned}
$$

It had proved in [9] that, in case $\widehat{d}(t)$ is estimated by (20)(21), UAD aspects are slowly time varying and $k_{0}=l_{4} / m_{u}-$ $l_{2} / m_{s}(t)>0$; then the following clause can be inferred: $\dot{e}(t)+$ $k_{0} e(t) \rightarrow 0 \forall \mathbf{x}(t)$. It means that $e(t) \rightarrow 0$; hence $\widehat{d}(t) \rightarrow d(t)$. Thus, based on Lemma 1 we can conclude that $S(t) \rightarrow 0$ is asymptotically stable in the sense of Lyapunov process. From (13) and (20)-(21), it can infer that the active force to control the MRD-based railway suspension can be separated into two parts as in (22), in which the main part, $u_{s}(t)$, comes from the Ad-op-SMC as in (23), while the second part, $u_{c}(t)$, is the supplement active force estimated by the NUO given in (24).

$$
\begin{aligned}
u(t)= & u_{s}(t)+u_{c}(t) \\
u_{s}(t)= & m_{s}(t) k_{1} x_{2}(t)-f_{s}(t)-f_{d}(t) \\
& +k_{2}\left(1-\exp \left(-k_{3}\left|x_{2}\right|\right)\right) m_{s}(t) \operatorname{sat}(S(t)) \\
& \\
u_{c}(t)= & \widehat{d}(t)
\end{aligned}
$$

In this paper, $k_{3}$ is chosen to be 0.3 .
4.3. Design of the T2F-SMC-TPM. Actually, it can be observed that the best SMC can be described via the main factors participating in the two following phases. From a sliding surface (SLS) depicting a stability norm, in the first phase, the system's dynamic response is controlled not only to direct towards the SLS but also to reach it as soon as possible. Then, as the second phase corresponding to the period when the system works on the SLS, system's dynamic response needs to be kept on moving along the sliding surface with an appropriate switching rate to stamp out the chattering phenomenon.

To establish an appropriate operating condition applying the above directions, in this paper, when the chassis mass and the track profile change, both the optimal slope $k_{1}$ of the SLS and adjusting velocity $k_{2}$ of control law (23) need to be adaptively interpolated. For this work, an IT-2FLS is used to identify the relation between the chassis mass, special features of the track profiles, and $k_{1}$ as well as $k_{2}$ via an experimental data set. As a result, the trained IT-2FLS called the T2F-SMCTPM is utilized to interpolate the optimal values of $k_{1}$ and $k_{2}$ which is then signed $\bar{k}_{1}$ and $\bar{k}_{2}$, respectively. By this way, the adaptively optimal SMC is created and called the Ad-opSMC. It should be noted that to set up the T2F-SMC-TPM, the train data set needs to be built which is presented as below.

In railway environment, real tracks are neither constantly straight nor perfect, and hence straights, curves, and track irregularities need to be integrated by different ways [29]. These track geometry features of track profiles impact directly on dynamic responses of the railway cars. In general, a real track profile is a summation of the isolated track geometry variations consisting of cusp, bump, jog, plateau, trough, sinusoid, and damped sinusoid with track irregularities [30]. The typical locations at which these profile types often occur have been also illustrated in this document. The bumpshaped profile with a disturbance surface expresses locations where two adjacent rails are to be connected (joints), or soft spots, washouts, mud spots, fouled ballast, spirals, grade crossings, bridges, overpasses, turnouts, or at the interlocking. The sinusoid profile expresses the track's uneven status at spirals, soft spots, or at bridges, while the track profile like trough with irregularities illustrates special areas such as soft spots and soft and unstable subgrades, or at spirals. Related to building the dataset for training the T2F-SMC-TPM, an issue here is how to model a track profile or, in general, what geometrical features can be used to express a track profile. In fact, there are several parameters expressing geometrical feature of a profile type. However, pragmatically, in this paper only parameters that could be estimated easily by an online measuring process are selected. They are the amplitude $(A)$ of the profile, the maximum of the power spectral density (PSD) of the track profile, and the peak frequency $\left(f^{\mathrm{PSD}}\right)$ when the PSD reaches the maximum value. For each track profile, the change level in the vertical displacement $x(t)$ in the time domain can be depicted by its amplitude while the frequency components of this signal can be illustrated via the power 
spectrum density of the time series $x(t)$. PSD describes how the power of the time series is distributed over the different frequencies. Hence, PSD can be used to estimate the bumpy status of the geometrical surface of the track profile.

Namely, normalized parameters of the maximum amplitude, the maximum of the PSD, the peak frequency, and the chassis mass, respectively, signed $\bar{A}, \overline{\mathrm{PSD}}, \bar{f}^{\mathrm{PSD}}$, and $\bar{m}_{s}$ are specified as follows. Regarding the $i$ th track profile, the normalized amplitude is defined as follows:

$$
\bar{A}_{i}=\frac{\left|A_{i}^{\max }-A_{i}^{\min }\right|}{2 A_{i}^{a}},
$$

where

$$
\begin{aligned}
A_{i}^{\max } & =\max _{j}\left\langle x_{i}^{j}\right\rangle ; \\
A_{i}^{\min } & =\min _{j}\left\langle x_{i}^{j}\right\rangle ; \\
A_{i}^{a} & =\frac{1}{Q_{i}} \sum_{j=1}^{Q_{i}}\left|x_{i}^{j}\right| .
\end{aligned}
$$

In the above, $x_{i}(t)=\left[x_{i}^{1}, \ldots, x_{i}^{\mathrm{Q}_{i}}\right]$ is the vertical displacement and $Q_{i}$ is the number of sampling points of the $i$ th track profile. For the $i$ th track profile, let $\mathrm{PSD}_{i}^{\max }$ and $\mathrm{PSD}_{i}^{a}$ be the maximum and average values of PSD, $p_{i}^{\text {non_zero }}$ be the set of $\operatorname{PSD}_{i}^{k}$ larger than a threshold value defined as $\varsigma_{p} \mathrm{PSD}_{i}^{\max }$, in which $\varsigma_{p}$ is a positive coefficient, chosen $\varsigma_{p}=0.01$ in this paper, and $l p_{i}^{\text {non_zero }}$ be the length of $p_{i}^{\text {non_zero }}$.

$$
\begin{aligned}
p_{i}^{\text {non_zero }} & =\left\{\operatorname{PSD}_{i}^{k} \mid \operatorname{PSD}_{i}^{k} \geq \varsigma_{p} \operatorname{PSD}_{i}^{\max }\right\}, \\
\operatorname{PSD}_{i}^{a} & =\sum_{k=1}^{l p_{i}^{\text {non_zero }}} \frac{\operatorname{PSD}_{i}^{k}}{l p_{i}^{\text {non_zero }}} .
\end{aligned}
$$

The normalized PSD is then defined as in (28).

$$
\overline{\operatorname{PSD}_{i}}=\frac{\operatorname{PSD}_{i}^{\max }}{\operatorname{PSD}_{i}^{a}} .
$$

Finally, for the $i$ th track profile, let $f_{i}^{\mathrm{PSD}}$ be the frequency corresponding to the maximum value of PSD, to say $\mathrm{PSD}_{i}^{\max }$; let $m_{s \_}$be the normal chassis mass; the normalized peak frequency and normalized mass are then defined as follows:

$$
\begin{aligned}
\bar{f}_{i}^{\mathrm{PSD}} & =\frac{f_{i}^{\mathrm{PSD}}}{\varsigma_{f} F_{s}} \\
\bar{m}_{s} & =\frac{m_{s}(t)}{m_{s \_n}},
\end{aligned}
$$

where $\varsigma_{f}$ is a positive coefficient, which is chosen $\varsigma_{f}=0.01$ in this paper, while $F_{s}$ is the surveyed frequency range.
Thus, the dataset for training the T2F-SMC-TPM is structured by the data samples typed 〈[input] - [output]〉 as follows:

$$
\left\langle\left[\bar{A}_{i}, \overline{\mathrm{PSD}}_{i}, \bar{f}_{i}^{\mathrm{PSD}}, \bar{m}_{s_{-} i}\right]-\left[\bar{k}_{1}, \bar{k}_{2}\right]_{i}\right\rangle, \quad i=1 \cdots P,
$$

where $P$ is the number of surveyed track profiles and chassis mass values.

The dataset (31) is then used to train the T2F-SMC-TPM using the algorithm OD-T2FLS [7]. Based on the established T2F-SMC-TPM, corresponding to the track profile features and the chassis mass at the sampling time, the two adaptively optimal parameters $\left[\bar{k}_{1}, \bar{k}_{2}\right]$ are interpolated. As a result, the main part (23) of the control damping force is finally calculated as below:

$$
\begin{aligned}
u_{s}(t)= & m_{s}(t) \bar{k}_{1} x_{2}(t)-f_{s}(t)-f_{d}(t) \\
& +\bar{k}_{2}\left(1-\exp \left(-k_{3}\left|x_{2}\right|\right)\right) m_{s}(t) \operatorname{sat}(S(t))
\end{aligned}
$$

In this study, $k_{3}$ is chosen by 0.3 .

In order to generate the required damping force, $f_{\mathrm{MR}}(t) \equiv$ $u(t), u(t)=u_{s}(t)+u_{c}(t)$, to control the suspension stamping out chassis vibration as shown in Figures 4 and 5, the inverse MRD model T2F-I-MRD for calculating the corresponding current $I(t)$ is built via a measured data set expressing dynamic response of the MRD. Based on the dataset and the algorithm OD-T2FLS [7], the T2F-I-MRD is then trained. This content is detailed in the next section.

\section{Experiment and Estimation}

5.1. Establishing the T2F-I-MRD. In order to build the T2FI-MRD, an experimental apparatus is established as shown in Figure 6. The load cell (3) is fixed to the piston shaft (6) of the MRD and directed by the upper bed (7) to connect to the shaft of the DC servo motor (2) via the translating rod (8). The cylinder of the MRD (4) is fixed to the lower bed (5). Based on the eccentric equipment (1), rotation of the motor shaft is transformed into translation motion of the damper piston. The signal from the load cell, MRD force $f_{\mathrm{MR}}$, is sent to the computer via the A/D converter and signal process equipment. By adjusting rotation velocity $\omega$ of the DC motor via the computer and motor driver and by adjusting the current $I$ applied to the MRD via the computer, the $\mathrm{D} / \mathrm{A}$ converter, and the amplifier, the corresponding $f_{\mathrm{MR}}$ is obtained. The input-output of the data set for building T2FI-MRD is $\left[\begin{array}{lll}x_{i 1} & x_{i 2} & x_{i 3}\end{array}\right] \equiv\left[\begin{array}{lll}d_{r e_{i}} & v_{r e_{i}} & f_{\mathrm{MR}_{i}}\end{array}\right]$ and $y_{i} \equiv I_{i}$, $i=1 \cdots P$, respectively, in which $P$ is the number of data samples.

5.2. Building the T2F-SMC-TPM. As mentioned in [30], a track profile is integrated by several typical track types such as the cusp, bump, jog, trough, plateau, sinusoid, and rail joint areas. Therefore we utilize a mathematical model depicting an uneven status with a disturbance 
surface as in (33) to build the database for training the T2F-SMC-TPM.

$$
x_{0}(t)= \begin{cases}0.5 X_{0}(1-\cos (\omega t)) & \text { if } 0 \leq t \leq \frac{T}{2} \text { or } \frac{3 T}{2} \leq t \leq 2 T \\ X_{0}(1+3 r) & \text { if } \frac{T}{2}<t<\frac{3 T}{2} \\ X_{0}(\sin (0.03 \omega t)+\sin (0.2 \omega t))+3 r X_{0} & \text { if } t>2 T .\end{cases}
$$

In (33), $\omega[\mathrm{rad} / \mathrm{s}]$ and $T=2 \pi / \omega=V / \bar{k}_{0}[\mathrm{~s}]$, respectively, are the angular frequency and cycle of the surveyed sinetyped road profile; $V$ is proportional rate to be chosen equal to the velocity $[\mathrm{m} / \mathrm{s}]$ of the train in this survey; $r=$ $\mid$ random $(0,0.05) \mid$ is a positive value chosen randomly around zero with a radius of $0.05 ; X_{0}=|\operatorname{random}(0,0.01)| \leq 0.01[\mathrm{~m}]$ is the amplitude of the signal which also is chosen randomly such that it distributes around zero with a radius of 0.01 ; $\bar{k}_{0}$ is a positive coefficient chosen randomly. In this paper, it belongs to $\bar{k}_{0} \in\left[\begin{array}{ll}5 & 15\end{array}\right]$. For example, by choosing $\bar{k}_{0}=$ 10 , changing the velocity from $10 \mathrm{~km} / \mathrm{h}$ to $300 \mathrm{~km} / \mathrm{h}$, and changing the sprung mass from $19,600 \mathrm{~kg}$ to $22,600 \mathrm{~kg}$, some extractions from the obtained track profiles are shown in Figure 7; deriving from the track profiles, based on the DE algorithm [28] and the proposed method with the principle parameters of the suspension given in Table 1, we obtained 30 input-output data samples as in Tables 2(a) and 2(b). By this way, the training database consisting of $P_{0}$ input-output data samples as mentioned above is generated. Based on the database and the algorithm OD-T2FLS [7], the T2F-SMCTPM is established.

5.3. Surveys and Results. Using the mechanical frame of the suspension presented in Figure 3 and Table 1, four different suspension systems are established to evaluate control performance of the proposed controller AT2FC. In addition, for the comparison work, the controller NFSmUoC in [9] and the controller CO-FSMC in [31] are adopted. The first suspension system is the passive (or the uncontrolled suspension) while the second, third, and fourth are the individual integration of each of the three controllers NFSmUoC, CO-FSMC, and AT2FC into the suspension to set up three controllable suspension systems. The four suspension systems are all applied to estimate the proposed method. Two random changing factors reflecting external disturbances are imposed to the four suspension systems. The first random factor is the chassis mass $m_{s}(t)=9600 \pm 3000 \mathrm{~kg}$ and the second one is the unknown uneven surface and profile status of the three considered track types as shown at the end of this subsection.

In all surveys, the standard ISO 2631-1997 is referred as the ride comfort criteria. This standard is especially devoted to riding comfort in train and widely utilized to test the ride quality such as building criteria outlined in MSRP (Manual of Standards and Recommended Practices) of the Association of American Railroads. Maximum value of displacement and acceleration, $A_{d}$ and $A_{a}$ as well as their average absolute values, $\bar{d}$ and $\bar{a}$, are all considered as follows:

$$
\begin{aligned}
A_{d} & =\max _{i=1 \cdots P}\left|z_{s}^{i}\right| \\
A_{a} & =\max _{i=1 \cdots P}\left|\ddot{z}_{s}^{i}\right| \\
\bar{d} & =\frac{1}{P} \sum_{i=1}^{P}\left|z_{s}^{i}\right| ; \\
\bar{a} & =\frac{1}{P} \sum_{i=1}^{P}\left|\ddot{z}_{s}^{i}\right| .
\end{aligned}
$$

In (34) and (35), $i$ expresses $i$ th sample; $P$ is the number of samples; $z_{s}, \ddot{z}_{s}$, respectively, are vertical displacement and acceleration of the chassis mass. Besides, analyzing frequency from the acceleration signal is also considered.

As mentioned above, three typical track types consisting of the bump-typed track, sine-typed track, and the broken rail with unknown uneven surfaces are used.

For the tracks, we used the three track types consisting of the bump-typed track, sine-typed track, and the broken rail with unknown uneven surfaces.

The first track type is used to verify the ability to pass over a length of the bump-existed rail in the presence of external disturbance. The status shown in Figure 8 always appears in the railways $[30,32]$ which can be described mathematically as

$$
\begin{aligned}
& x_{0}(t) \\
& = \begin{cases}X_{0}\left[1-\cos \left(\omega_{r} t\right)\right]+\operatorname{rand}\left(0, d_{0}\right) & \text { if } \frac{2 \pi}{\omega_{r}} \leq t \leq \frac{4 \pi}{\omega_{r}} \\
\operatorname{rand}\left(0, d_{0}\right) & \text { otherwise. }\end{cases}
\end{aligned}
$$

In the above, $X_{0}$ is the half of the bump height; $\omega_{r}=2 \pi V_{c} / D$; $D$ is the bump width; $V_{c}$ is the train velocity passing over the bump. In the above, $\operatorname{rand}\left(0, d_{0}\right)$ is a number surrounding zero with an absolute amplitude smaller or equal to $d_{0}$ representing the uneven track surface. Here we use $X_{0}=$ $0.1 \mathrm{~m}, D=50 \mathrm{~m}, V_{c}=27.7778 \mathrm{~m} / \mathrm{s}(100 \mathrm{~km} / \mathrm{h})$, and $d_{0}=$ 0.002. By using (25), (28), and (29), the normalized GSFs are obtained as $\bar{A}=2.7806, \overline{\mathrm{PDS}}=2.0002$, and $\bar{f}^{\mathrm{PDS}}=4$, to which the T2F-SMC-TPM can interpolate the optimal parameters of the Ad-op-SMC based on $\bar{m}_{s}$ (30). For example, in this case its optimal parameters are $\bar{k}_{1}=675.1164$ and $\bar{k}_{2}=516.9222$ corresponding to $\bar{m}_{s}=1.038$. The survey 
TABLE 1: Parameters of the suspension system.

\begin{tabular}{lc}
\hline$m_{s}$ & $19600 \pm 3000 \mathrm{~kg}$ \\
$m_{u}$ & $1440 \mathrm{~kg}$ \\
$k_{s}$ & $392 \cdot 10^{4} \mathrm{~N} / \mathrm{m}$ \\
$k_{t}$ & $2804 \cdot 10^{4} \mathrm{~N} / \mathrm{m}$ \\
$c_{s}$ & $5.390 \cdot 10^{4} \mathrm{Ns} / \mathrm{m}$ \\
$c_{t}$ & $6.838 \cdot 10^{4} \mathrm{Ns} / \mathrm{m}$ \\
$l_{1}$ & 32 \\
$l_{2}$ & 12 \\
$l_{3}$ & 25 \\
$l_{4}$ & 350 \\
\hline
\end{tabular}

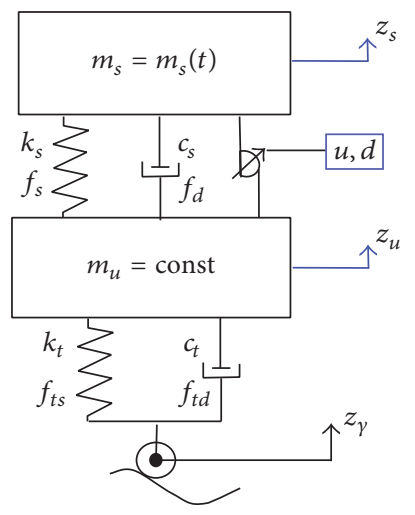

FIGURE 3: The active suspension system for the quarter car model.

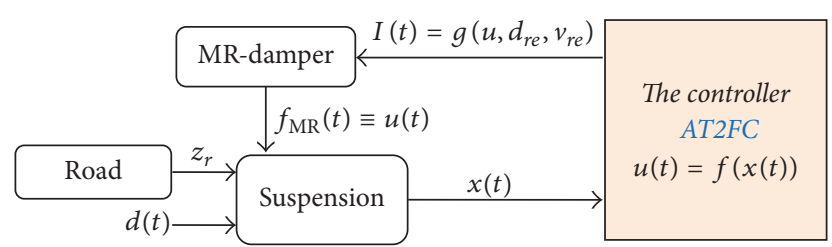

FIGURE 4: The MR-damper used as an actuator for the control strategy of the proposed active suspension controller AT2FC $\left(d_{r e}(t)=\right.$ $\left.x_{1}(t)-x_{3}(t) ; v_{r e}(t)=x_{2}(t)-x_{4}(t)\right)$.

results are then obtained as shown in Figures 9 and 10 and Table 3.

For the second rail status, a sine-typed track profile with a varying amplitude and cycle along with an uneven surface depicted by $\operatorname{rand}\left(0,0.5 d_{0}\right)$ is employed to express a train going up and down through a length of rail with bump-typed tracks. As mentioned in $[30,32,33]$, this track type often exists in the railways. For the one shown in Figure 11 with the amplitude of $0.0536(\mathrm{~m})$ and cycle of $1.2(\mathrm{sec})$ which describes a train with the speed of $50 \mathrm{~km} / \mathrm{h}$ to be traveling through the length, the normalized GSFs are obtained as follows: $\bar{A}=$ $2.6391, \overline{\mathrm{PDS}}=4.094$, and $\bar{f}^{\mathrm{PDS}}=18$. Therefore, by using the T2F-SMC-TPM, the Ad-op-SMC is interpolated; in case of $\bar{m}_{s}=1.1$, its optimal parameters are $\bar{k}_{1}=2419.2504$ and $\bar{k}_{2}=$ 1799.2112. Figures 12 and 13 and Table 4 illustrate the obtained survey results.

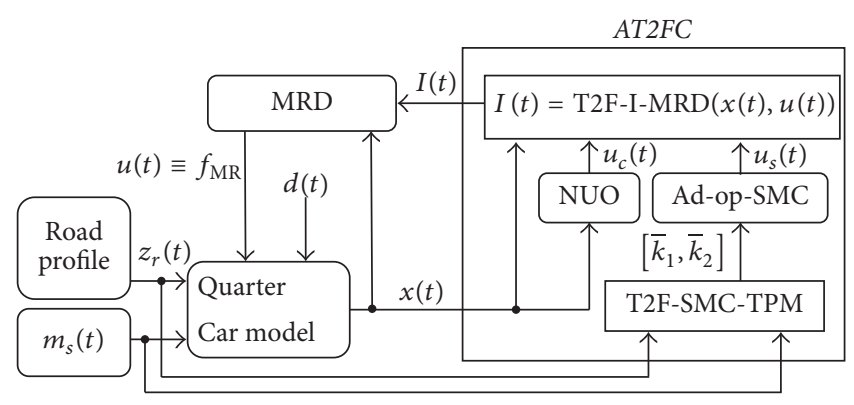

FIGURE 5: The structure and operating principle of the proposed controller AT2FC.

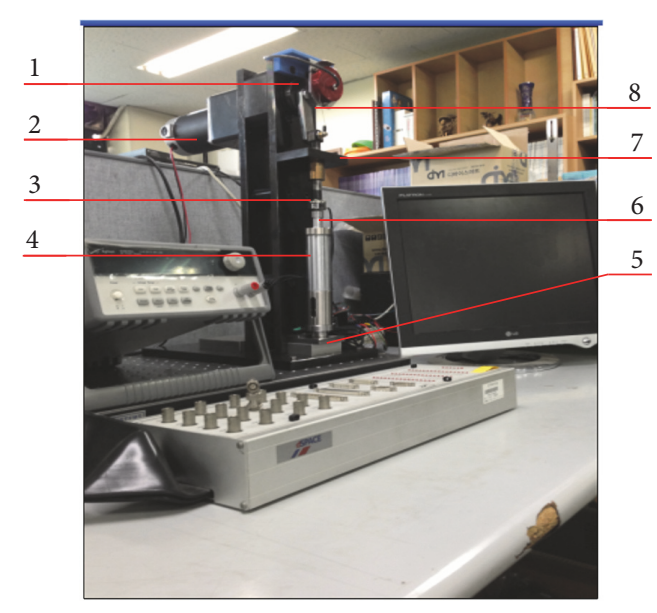

FIGURE 6: Experimental apparatus for building the dataset for training the T2F-I-MRD.

The other rail status related to a dangerous working condition of the system is also considered as a third case. Reality has shown that broken rails or welds or detail fractures are the main causes of derailment (http://safetydata.fra.dot .gov/), in which broken rails are the leading cause of the accident [33]. A broken rail with the disturbed surface shows in Figure 14(a) (https://www.networkrail.co.uk/running-therailway/looking-after-the-railway/delays-explained/). Figure 14(b) depicts train wheels operating with the velocity of $100 \mathrm{~km} / \mathrm{h}$ to be passing over the broken area at the instant $t=1$ second. In this case, we obtained $\bar{A}=69.2582, \overline{\mathrm{PSD}}=2.4559$, and $\bar{f}^{\text {PSD }}=9$. The T2F-SMC-TPM is then used to update the Ad-op-SMC. In this case of $\bar{m}_{s}=1.1$, Ad-op-SMC's optimal parameters are $\bar{k}_{1}=2783.15$ and $\bar{k}_{2}=2021.55$. The results obtained from this track profile are shown in Figures 15-17 and Table 5.

5.4. Discussion. The results obtained from the three track profiles reflect that the ability to stamp out vibration of the suspension controlled by the proposed controller AT2FC is better than that controlled by the NFSmUoC [9] or the COFSMC [31], and much better than the passive one. Firstly, it is seen from Figures 9, 12, and 15 and Tables 3-5 that, from the 
TABLE 2

(a) The dataset for building the T2F-SMC-TPM corresponding to $\bar{m}_{s}=\left[\begin{array}{ll}1.000 & 1.038\end{array}\right]$

\begin{tabular}{|c|c|c|c|c|c|c|c|c|c|}
\hline \multirow{2}{*}{ Number } & \multicolumn{3}{|c|}{ Special features } & \multirow{2}{*}{$\begin{array}{c}\text { Mass } \\
\bar{m}_{s}\end{array}$} & \multicolumn{2}{|c|}{ Optimal SMC } & \multirow{2}{*}{$\begin{array}{c}\text { Mass } \\
\bar{m}_{s}\end{array}$} & \multicolumn{2}{|c|}{ Optimal SMC } \\
\hline & $\bar{A}$ & $\overline{\mathrm{PSD}_{i}}$ & $\bar{f}^{\mathrm{PSD}}$ & & $\bar{k}_{1}$ & $\bar{k}_{2}$ & & $\bar{k}_{1}$ & $\bar{k}_{2}$ \\
\hline 1 & 8.5253 & 5.0648 & 14 & & 2335.0716 & 1822.7658 & & 953.2621 & 722.1579 \\
\hline 2 & 4.5223 & 3.3975 & 8 & & 906.5074 & 735.9054 & & 841.9140 & 659.5604 \\
\hline 3 & 8.7672 & 2.9489 & 6 & & 2540.8886 & 1982.8015 & & 990.1407 & 750.2305 \\
\hline 4 & 4.9732 & 5.4584 & 4 & & 711.9458 & 582.0642 & & 758.5645 & 596.0658 \\
\hline 5 & 2.0376 & 3.8494 & 4 & & 2142.8384 & 1673.2942 & & 2034.4626 & 1530.7845 \\
\hline 6 & 1.2514 & 4.1052 & 2 & & 904.2789 & 712.2077 & & 923.4180 & 721.6689 \\
\hline 7 & 0.9223 & 3.9994 & 2 & & 2133.5566 & 1666.7040 & & 2422.9525 & 1821.3986 \\
\hline 8 & 0.8066 & 3.1138 & 2 & & 865.9687 & 680.8311 & & 890.6681 & 696.9541 \\
\hline 9 & 1.3385 & 3.3878 & 2 & & 840.4636 & 658.2600 & & 2190.2782 & 1647.6059 \\
\hline 10 & 1.5627 & 3.8997 & 2 & & 983.9933 & 798.1658 & & 2713.4929 & 2038.7391 \\
\hline 11 & 1.6466 & 2.6311 & 6 & & 629.8220 & 515.9764 & & 626.9616 & 482.9901 \\
\hline 12 & 1.6149 & 2.3337 & 2 & & 4169.1999 & 3000.3139 & & 2713.8037 & 482.9901 \\
\hline 13 & 1.4922 & 2.8278 & 2 & & 919.8343 & 721.0951 & & 2702.1637 & 2030.3610 \\
\hline 14 & 1.4764 & 2.5080 & 2 & & 2612.6371 & 1345.8986 & & 2288.6442 & 1720.3469 \\
\hline 15 & 1.5229 & 3.0562 & 2 & 1000 & 945.9174 & 743.7041 & 1038 & 989.4768 & 748.8851 \\
\hline 16 & 1.6485 & 3.5864 & 2 & & 944.8328 & 740.8054 & 1.038 & 2812.7509 & 1394.6906 \\
\hline 17 & 1.7683 & 4.7461 & 4 & & 2890.0544 & 2253.6125 & & 2926.9739 & 2198.3221 \\
\hline 18 & 1.8755 & 4.9534 & 2 & & 2832.5362 & 2208.8614 & & 2099.8805 & 1579.3051 \\
\hline 19 & 1.9866 & 5.1213 & 2 & & 904.9660 & 710.6018 & & 910.8603 & 690.5929 \\
\hline 20 & 1.7298 & 4.2015 & 2 & & 2193.6037 & 1713.3309 & & 2591.9169 & 1948.0186 \\
\hline 21 & 1.8413 & 4.2470 & 2 & & 879.6654 & 691.2135 & & 3074.3839 & 2133.4457 \\
\hline 22 & 1.8428 & 3.6829 & 4 & & 1250.6077 & 979.5402 & & 2577.5019 & 1936.7388 \\
\hline 23 & 2.0466 & 3.7993 & 4 & & 2621.1654 & 2045.2430 & & 2479.5176 & 1863.9505 \\
\hline 24 & 1.8469 & 3.8806 & 2 & & 3040.0223 & 2369.7382 & & 2771.7986 & 2082.1558 \\
\hline 25 & 1.9317 & 4.1203 & 4 & & 2107.6196 & 1645.9793 & & 2442.4745 & 1836.2908 \\
\hline 26 & 1.7797 & 3.7806 & 4 & & 2381.1191 & 1858.3251 & & 902.6679 & 705.8501 \\
\hline 27 & 1.7707 & 3.2965 & 6 & & 2711.6028 & 2113.0585 & & 3022.1381 & 2269.1541 \\
\hline 28 & 1.5443 & 3.7395 & 4 & & 758.8964 & 618.6243 & & 773.0376 & 612.3174 \\
\hline 29 & 1.6267 & 4.1595 & 4 & & 798.8963 & 654.6223 & & 799.0375 & 666.3177 \\
\hline 30 & 1.5219 & 3.6662 & 4 & & 2204.1198 & 1721.1770 & & 994.7896 & 752.4646 \\
\hline
\end{tabular}

(b) The dataset for building the T2F-SMC-TPM corresponding to $\bar{m}_{s}=\left[\begin{array}{lll}1.077 & 1.115 & 1.153\end{array}\right]$

\begin{tabular}{|c|c|c|c|c|c|c|c|c|c|}
\hline \multirow{2}{*}{ Number } & \multirow{2}{*}{$\begin{array}{c}\text { Mass } \\
\bar{m}_{s}\end{array}$} & \multicolumn{2}{|c|}{ Optimal SMC } & \multirow{2}{*}{$\bar{m}_{s}$} & \multicolumn{2}{|c|}{ Optimal SMC } & \multirow{2}{*}{$\begin{array}{c}\text { Mass } \\
\bar{m}_{s}\end{array}$} & \multicolumn{2}{|c|}{ Optimal SMC } \\
\hline & & $\bar{k}_{1}$ & $\bar{k}_{2}$ & & $\bar{k}_{1}$ & $\bar{k}_{2}$ & & $\bar{k}_{1}$ & $\bar{k}_{2}$ \\
\hline 1 & & 922.5776 & 675.2737 & & 2229.1609 & 1561.5576 & & 2133.2607 & 1445.4078 \\
\hline 2 & & 871.3061 & 638.8431 & & 832.0436 & 590.2148 & & 1912.2503 & 1296.9057 \\
\hline 3 & & 2568.7236 & 1862.4014 & & 3457.9600 & 2233.9507 & & 2375.6936 & 1608.9346 \\
\hline 4 & & 787.6981 & 596.5456 & & 744.4079 & 528.6801 & & 714.6500 & 506.9179 \\
\hline 5 & & 2159.8919 & 1567.2162 & & 847.4220 & 600.1297 & & 2299.7221 & 1027.3687 \\
\hline 6 & & 2695.6631 & 1953.3682 & & 891.4124 & 651.0358 & & 2487.3345 & 1684.2294 \\
\hline 7 & & 2789.9277 & 2021.9472 & & 2585.3534 & 1810.2371 & & 2885.3419 & 1803.9449 \\
\hline 8 & & 2436.4373 & 1766.8375 & & 908.0902 & 642.3536 & & 1775.0764 & 1204.6414 \\
\hline 9 & & 2147.1477 & 1558.5583 & & 872.3525 & 638.8025 & & 870.2371 & 596.0257 \\
\hline 10 & & 2571.4401 & 1864.0762 & & 2789.9754 & 1952.1905 & & 5713.8451 & 3564.1694 \\
\hline 11 & & 631.0044 & 480.7390 & & 660.0787 & 441.5234 & & 614.4037 & 439.0579 \\
\hline 12 & & 2601.8914 & 1885.4678 & & 3084.4082 & 2157.4933 & & 2985.9725 & 2019.9774 \\
\hline 13 & & 5273.5119 & 2513.4696 & & 2644.7622 & 1223.3106 & & 3181.9203 & 1988.6962 \\
\hline 14 & & 2540.8062 & 1841.7282 & & 2502.0637 & 1751.1615 & & 2563.7333 & 1735.0979 \\
\hline 15 & 1077 & 2458.2745 & 1782.3050 & 1115 & 4259.0243 & 2749.8682 & 1153 & 2528.2212 & 1711.6512 \\
\hline 16 & & 3304.1382 & 2210.6709 & & 2407.7749 & 1686.1397 & & 2262.2689 & 1532.3271 \\
\hline
\end{tabular}


(b) Continued.

\begin{tabular}{|c|c|c|c|c|c|c|c|c|c|}
\hline \multirow{2}{*}{ Number } & \multirow{2}{*}{$\begin{array}{c}\text { Mass } \\
\bar{m}_{s}\end{array}$} & \multicolumn{2}{|c|}{ Optimal SMC } & \multirow{2}{*}{$\bar{m}_{s}$} & \multicolumn{2}{|c|}{ Optimal SMC } & \multirow{2}{*}{$\begin{array}{c}\text { Mass } \\
\bar{m}_{s}\end{array}$} & \multicolumn{2}{|c|}{ Optimal SMC } \\
\hline & & $\bar{k}_{1}$ & $\bar{k}_{2}$ & & $\bar{k}_{1}$ & $\bar{k}_{2}$ & & $\bar{k}_{1}$ & $\bar{k}_{2}$ \\
\hline 17 & & 2877.0359 & 2084.1402 & & 2785.3674 & 1949.1730 & & 2567.3645 & 1737.7531 \\
\hline 18 & & 2613.6180 & 1749.5122 & & 2349.6060 & 1645.9095 & & 2825.7581 & 1911.7728 \\
\hline 19 & & 2484.8961 & 1189.0543 & & 2259.4447 & 1583.1771 & & 2889.1311 & 1806.4919 \\
\hline 20 & & 2634.3620 & 1908.7435 & & 2741.9918 & 1918.9206 & & 3082.0398 & 2084.7294 \\
\hline 21 & & 2032.3262 & 1475.2905 & & 891.2040 & 629.9354 & & 2940.6243 & 1837.3593 \\
\hline 22 & & 2236.4723 & 1622.8059 & & 2796.8879 & 1957.4390 & & 2918.0208 & 1973.8165 \\
\hline 23 & & 2770.3559 & 2007.3993 & & 2696.2571 & 1887.2921 & & 3192.4595 & 1995.1240 \\
\hline 24 & & 1939.3980 & 1408.2689 & & 2067.7597 & 1449.3634 & & 2667.9370 & 1806.0082 \\
\hline 25 & & 2256.4241 & 1636.3654 & & 2346.4782 & 1644.1093 & & 2595.5186 & 1757.2010 \\
\hline 26 & & 2398.4572 & 1739.2731 & & 2388.5288 & 1673.1055 & & 2368.3608 & 1604.3651 \\
\hline 27 & & 3069.3155 & 2222.8377 & & 3305.5004 & 2311.3287 & & 2863.3061 & 1937.0000 \\
\hline 28 & & 1597.3052 & 1122.2111 & & 779.7302 & 571.8900 & & 777.9218 & 534.3333 \\
\hline 29 & & 1556.3052 & 1131.2191 & & 778.7302 & 571.8907 & & 750.9214 & 569.3341 \\
\hline 30 & & 2343.1691 & 1699.9124 & & 1964.3744 & 1377.3736 & & 2642.7485 & 1788.9367 \\
\hline
\end{tabular}

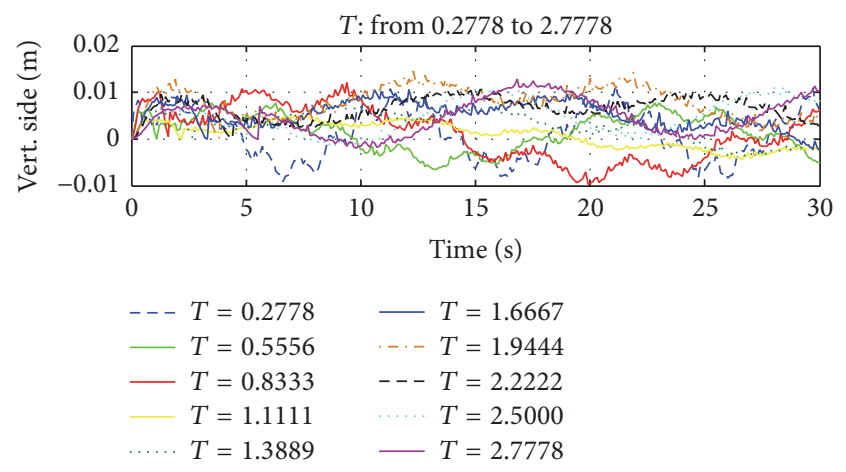

(a)

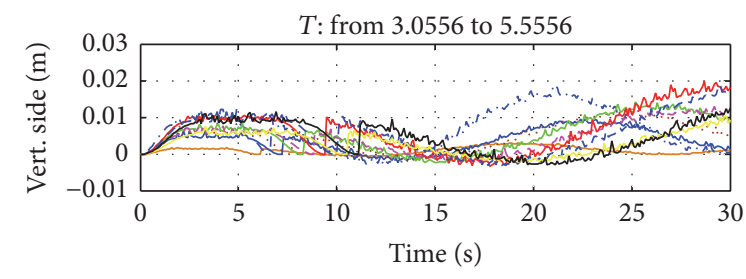

$\begin{aligned}-T & =3.0556 \\ -T & =3.3333 \\ T & =3.6111 \\ \cdots T & =3.8889 \\ -T & =4.1667\end{aligned}$

$---T=4.4444$

$-T=4.7222$

$--T=5$

$T=5.2778$

$-T=5.5556$

(b)

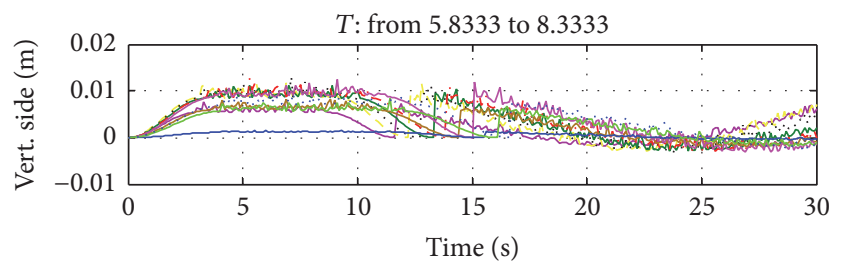

$$
\begin{aligned}
-T & =5.8333 & -T & =7.2222 \\
T & =6.1111 & -T & =7.5000 \\
\ldots . T & =6.3889 & -T & =7.7778 \\
-T & =6.6667 & -T & =8.0556 \\
-T & =6.9444 & \cdots . T & =8.3333
\end{aligned}
$$

(c)

Figure 7: Track profiles are used to build the dataset for establishing the T2F-SMC-TPM in which T is adjusted from 0.2778 to 2.7778 second (a), 3.0556 to 5.5556 second (b), and 5.8333 to 8.3333 second (c).

chassis displacement responses, there is a contrast between the result from the AT2FC and the passive system; the difference between chassis displacements among the controlled ones decreases significantly; however the lowest value belongs to the AT2FC. For example, $A_{d}(34)$ corresponding to the AT2FC, CO-FSMC, and the passive, respectively, are $0.0269,0.0617$, and $0.1616(\mathrm{~m})$ for the bump-typed track, or $0.0084,0.0094$, and $0.0368(\mathrm{~m})$ for the sine-typed track. In a similar fashion, for the broken rail case, $A_{d}$ coming from the AT2FC, NFSmUoC, and the passive are $1.4463 .10^{-4}$, $1.5713 .10^{-4}$, and $0.0024(\mathrm{~m})$, respectively. Regarding chassis acceleration, the qualitative illustration shown in Figures 10, 13 , and 16 as well as the quantitative estimation given in Tables 3-5 indicate that the AT2FC is more effective than COFSMC and NFSmUoC, and much better than the passive one. For example, for the sine-typed track, $A_{a}(34)$ related to the 


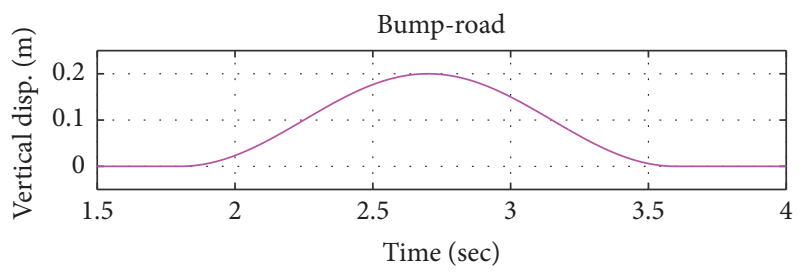

FIGURE 8: The bump-typed track profile is used to estimate the AT2FC.

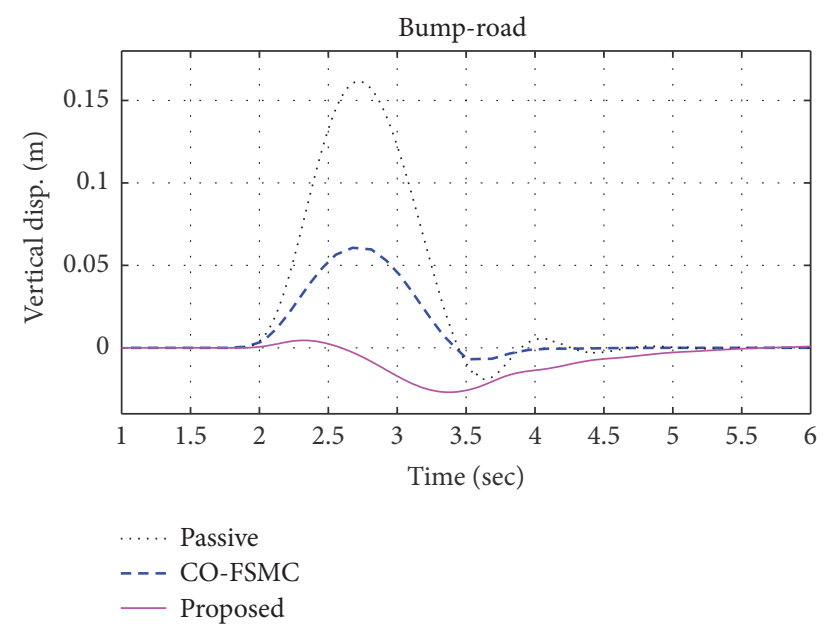

Figure 9: The bump-typed track profile: vertical chassis displacement corresponding to each method.

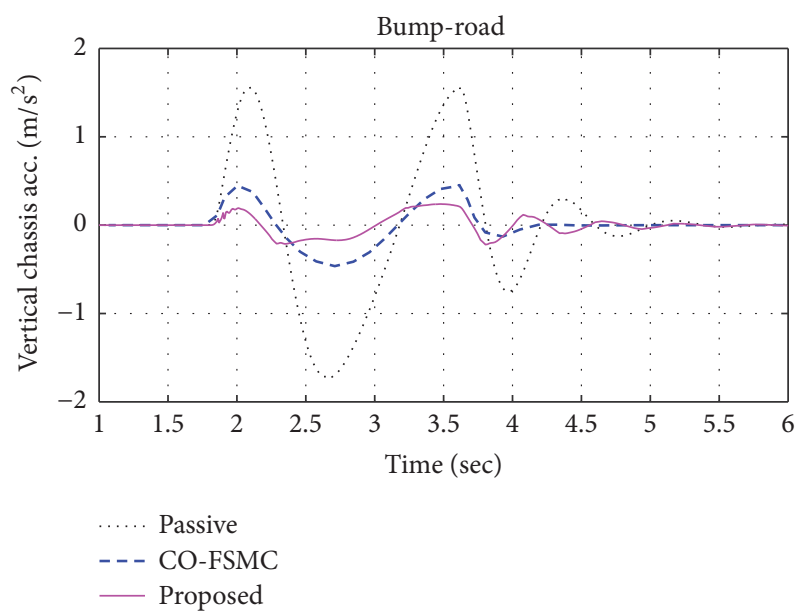

FIGURE 10: The bump-typed track profile: vertical chassis acceleration corresponding to each method.

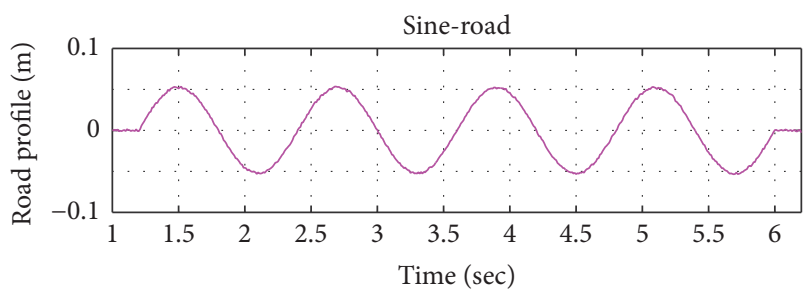

FIGURE 11: The sine-typed track profile is used to estimate the AT2FC.

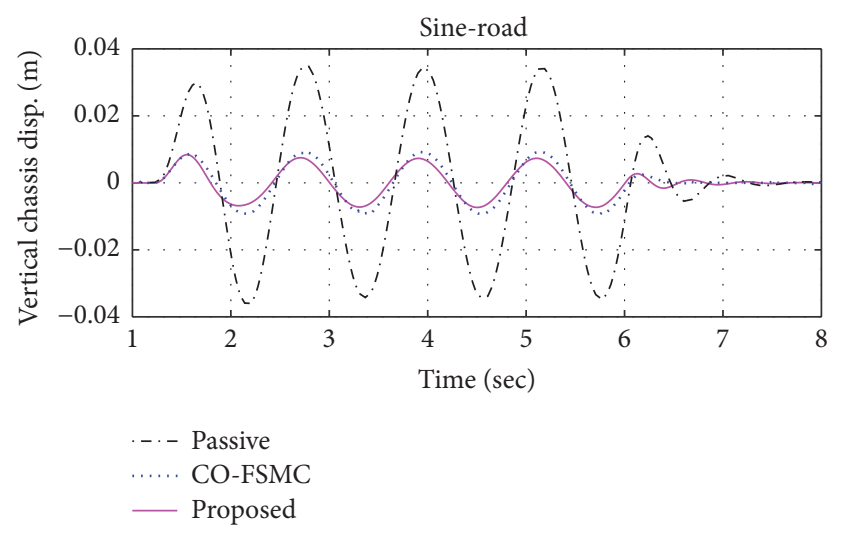

FIGURE 12: The sine-type track profile: vertical chassis displacement corresponding to each method.

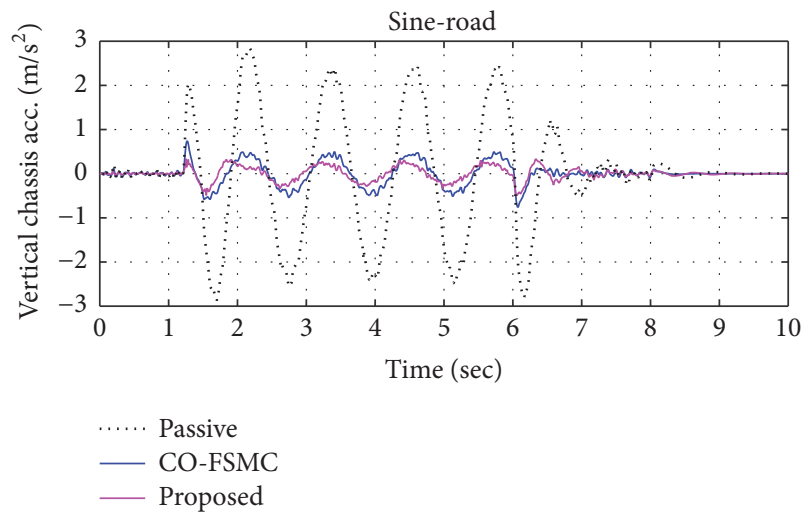

FIGURE 13: The sine-type track profile: vertical chassis acceleration corresponding to each method.

TABLE 3: The bump-typed track profile: vertical chassis displacement and acceleration.

\begin{tabular}{lcccc}
\hline & \multicolumn{2}{c}{ Displacement $(\mathrm{mm})$} & \multicolumn{2}{c}{ Acceleration $\left(\mathrm{m} / \mathrm{s}^{2}\right)$} \\
& $A_{d}$ & $\bar{d}$ & $A_{a}$ & $\bar{a}$ \\
\hline Passive & 0.1616 & 0.0138 & 1.7313 & 0.2285 \\
CO-FSMC & 0.0617 & 0.0054 & 0.4772 & 0.0605 \\
Proposed & 0.0269 & 0.0065 & 0.2390 & 0.0764 \\
\hline
\end{tabular}

TABLE 4: The sine-typed track profile: vertical chassis displacement and acceleration.

\begin{tabular}{lcccc}
\hline & \multicolumn{2}{c}{ Displacement $(\mathrm{mm})$} & \multicolumn{2}{c}{ Acceleration $\left(\mathrm{m} / \mathrm{s}^{2}\right)$} \\
& $A_{d}$ & $\bar{d}$ & $A_{a}$ & $\bar{a}$ \\
\hline Passive & 0.0368 & 0.0137 & 2.8684 & 0.5892 \\
CO-FSMC & 0.0094 & 0.0036 & 0.7612 & 0.1097 \\
Proposed & 0.0084 & 0.0027 & 0.4978 & 0.0966 \\
\hline
\end{tabular}

AT2FC, CO-FSMC, and the passive are 0.4978, 0.7612, and $2.8684\left(\mathrm{~m} / \mathrm{s}^{2}\right)$; for the broken rail case, $A_{a}$ corresponding to the AT2FC, NFSmUoC and the passive are 1.3348, 1.8530, and $4.0969 \mathrm{~m} / \mathrm{s}^{2}$, while $\bar{a}$ (35) for the broken rail case deriving from the AT2FC, NFSmUoC, and the passive are 0.0515, 


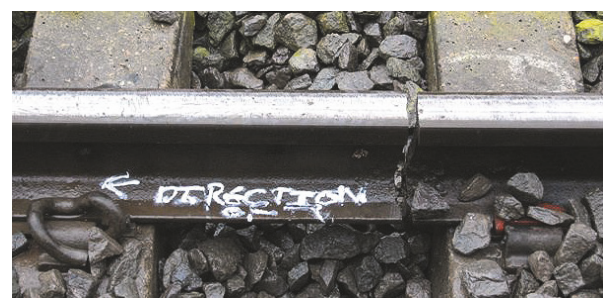

(a)

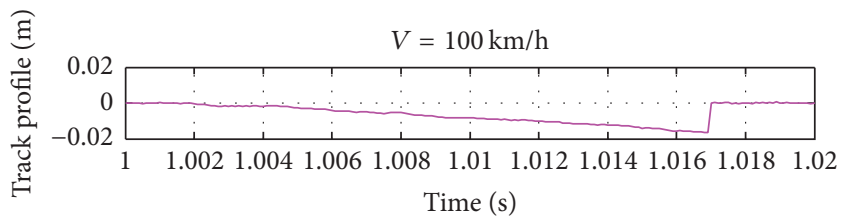

(b)

FIGURE 14: Broken rail with a disturbance surface: the real picture of the track profile (a) and the model when a train passes over it with a velocity of $100 \mathrm{~km} / \mathrm{h}(\mathrm{b})$.

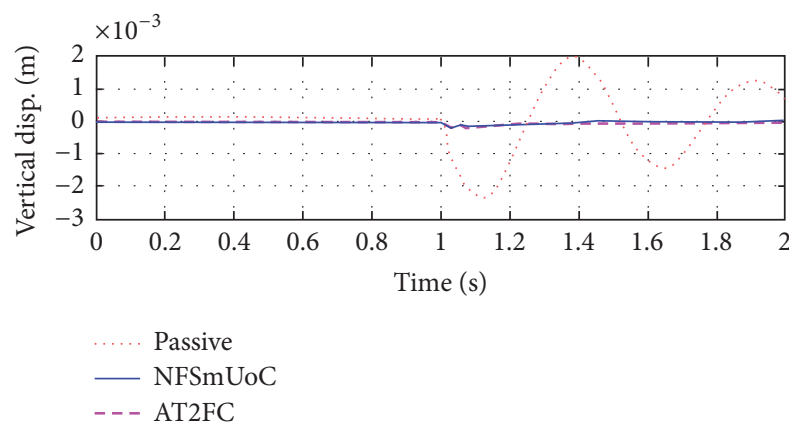

FIGURE 15: Vertical displacement related to each control method.

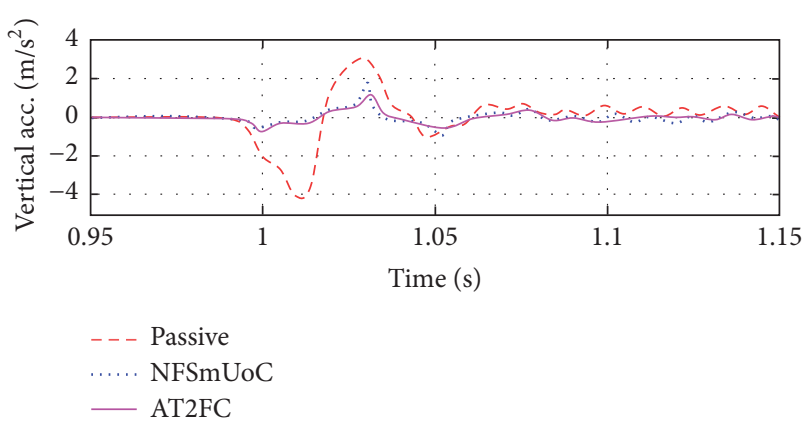

FIGURE 16: Vertical acceleration related to each control method.

0.0830 , and $0.1651 \mathrm{~m} / \mathrm{s}^{2}$, respectively. Although in some cases $\bar{d}$ and $\bar{a}$ coming from the AT2FC are slightly higher than those from the NFSmUoC or CO-FSMC, the AT2FC is the most effective one by means of the ride comfort criteria because the dependence of the criteria on $A_{d}$ and $A_{a}$ is more considerable than that on $\bar{d}$ and $\bar{a}$.

Related to the frequency domain, analyzing power spectral density (PSD) of the acceleration signal is also considered as shown in Figure 17. Being much the same as the corresponding results obtained in the time domain, PSD of acceleration of the chassis vibration controlled by the AT2FC is smaller than that controlled by the NFSmUoC and much smaller than PSD of acceleration from the passive one. Besides, although all of the three methods exist resonance zones, the maximum value of PSD in these zones related to the proposed controller is the smallest.

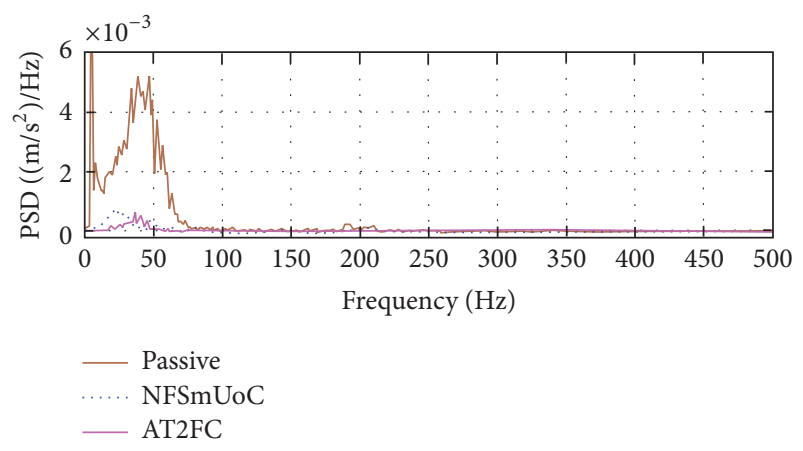

FIGURE 17: PSD of the acceleration signal of the three suspension systems.

TABLE 5: Dynamic response of $m_{s}$ corresponding to the broken rail with a disturbance surface.

\begin{tabular}{lcccc}
\hline & \multicolumn{2}{c}{ Displacement $(\mathrm{mm})$} & \multicolumn{2}{c}{ Acceleration $\left(\mathrm{m} / \mathrm{s}^{2}\right)$} \\
& $A_{d}$ & $\bar{d}$ & $A_{a}$ & $\bar{a}$ \\
\hline Passive & 0.0024 & $5.3920 \cdot 10^{-4}$ & 4.0969 & 0.1651 \\
NFSmUoC & $1.5713 \cdot 10^{-4}$ & $2.3083 \cdot 10^{-5}$ & 1.8530 & 0.0830 \\
Proposed & $1.4463 \cdot 10^{-4}$ & $3.1332 \cdot 10^{-5}$ & 1.3348 & 0.0515 \\
\hline
\end{tabular}

Another aspect can be also seen from Tables 2(a) and 2(b) that the optimal structure of the SMC is sensitive not only to the change of the sprung mass $m_{s}(t)$ but also to the special features of the track profiles. When the load and GSFs change, the optimal structure of the SMC depicted by $\bar{k}_{1}, \bar{k}_{2}$ is changed in a quite wide range. As a result, if the train's operating condition changes quickly, the ability to adapt of the original SMC becomes worse remarkably. This is the main reason why the Ad-op-SMC should be used instead of the original SMC.

\section{Conclusion}

In this work, a new adaptive type 2 fuzzy sliding controller (AT2FC) for vibration control of MRD-based railway suspension systems has been developed and successfully implemented via experimental realization. In order to cope with UAD existing in real operation conditions, the proposed controller has been formulated by establishing the adaptive 
ability. The AT2FC consists of four main parts: the Ad-opSMC, T2F-SMC-TPM, NUO, and the T2F-I-MRD. In the operating process, via the T2F-SMC-TPM, the Ad-op-SMC is updated by the adaptively optimal parameters according as the real time status of the load and track profile. By using the Ad-op-SMC and NUO, the error between the required damping force and the corresponding estimated one is improved. As a result, by using the T2F-I-MRD and the estimated damping force, the input current for the MRD is specified to stamp out chassis vibration more effectively. Together with the stability attribute proved theoretically, it has been also verified from the surveys that the proposed AT2FC can provide an adaptive ability against UAD better than the previous control methods.

\section{Conflicts of Interest}

The authors declare that there are no conflicts of interest regarding the publication of this paper.

\section{Acknowledgments}

This work was supported by Inha University Research Grant in 2016.

\section{References}

[1] R. Adhikari and H. Yamaguchi, "Sliding mode control of buildings with ATMD," Earthquake Engineering and Structural Dynamics, vol. 26, no. 4, pp. 409-422, 1997.

[2] S. D. Nguyen and T. Seo, "Establishing ANFIS and the use for predicting sliding control of active railway suspension systems subjected to uncertainties and disturbances," International Journal of Machine Learning and Cybernetics.

[3] J. C. Wu, J. N. Yang, and A. K. Agrawal, "Applications of sliding mode control to benchmark problems," Earthquake Engineering and Structural Dynamics, vol. 27, no. 11, pp. 1247-1265, 1998.

[4] J.-J. E. Slotine, "Sliding mode controller design for non-linear systems," International Journal of Control, vol. 40, no. 2, pp. 421434, 1984.

[5] V. S. Deshpande, P. D. Shendge, and S. B. Phadke, "Active suspension systems for vehicles based on a sliding-mode controller in combination with inertial delay control," Proceedings of the Institution of Mechanical Engineers, Part D: Journal of Automobile Engineering, vol. 227, no. 5, pp. 675-690, 2013.

[6] S. D. Nguyen, S. Choi, and T. Seo, "Recurrent Mechanism and Impulse Noise Filter for Establishing ANFIS," IEEE Transactions on Fuzzy Systems, pp. 1-1.

[7] S. D. Nguyen, S.-B. Choi, and Q. H. Nguyen, "An optimal design of interval type-2 fuzzy logic system with various experiments including magnetorheological fluid damper," Proceedings of the Institution of Mechanical Engineers, Part C: Journal of Mechanical Engineering Science, vol. 228, no. 17, pp. 3090-3106, 2014.

[8] S. D. Nguyen, Q. H. Nguyen, and T.-I. Seo, "ANFIS deriving from jointed input-output data space and applying in smartdamper identification," Applied Soft Computing Journal, vol. 53, pp. 45-60, 2017.
[9] S. D. Nguyen and Q. H. Nguyen, "Design of active suspension controller for train cars based on sliding mode control, uncertainty observer and neuro-fuzzy system," Journal of Vibration and Control, vol. 23, no. 8, pp. 1334-1353, 2017.

[10] J. M. Mendel, R. I. John, and F. Liu, "Interval type-2 fuzzy logic systems made simple," IEEE Transactions on Fuzzy Systems, vol. 14, no. 6, pp. 808-821, 2006.

[11] O. Castillo and P. Melin, "A review on the design and optimization of interval type-2 fuzzy controllers," Applied Soft Computing Journal, vol. 12, no. 4, pp. 1267-1278, 2012.

[12] M. H. Khooban, T. Niknam, F. Blaabjerg, and M. Dehghani, "Free chattering hybrid sliding mode control for a class of nonlinear systems: electric vehicles as a case study," IET Science, Measurement and Technology, vol. 10, no. 7, pp. 776-785, 2016.

[13] M. H. Khooban, T. Niknam, and M. Sha-Sadeghi, "Speed control of electrical vehicles: a time-varying proportional-integral controller-based type-2 fuzzy logic," IET Science, Measurement \& Technology, vol. 10, no. 3, pp. 185-192, 2016.

[14] V. S. Deshpande, M. Bhaskara, and S. B. Phadke, "Sliding mode control of active suspension systems using a disturbance observer," in Proceedings of the 12th International Workshop on Variable Structure Systems (VSS '12), pp. 70-75, 2012.

[15] M. J. Corless and G. Leitmann, "Continuous state feedback guaranteeing uniform ultimate boundedness for uncertain dynamic systems," Institute of Electrical and Electronics Engineers. Transactions on Automatic Control, vol. 26, no. 5, pp. 11391144, 1981.

[16] V. S. Deshpande, B. Mohan, P. D. Shendge, and S. B. Phadke, "Disturbance observer based sliding mode control of active suspension systems," Journal of Sound and Vibration, vol. 333, no. 11, pp. 2281-2296, 2014.

[17] W.-H. Chen, "Nonlinear disturbance observer-enhanced dynamic inversion control of missiles," Journal of Guidance, Control, and Dynamics, vol. 26, no. 1, pp. 161-166, 2003.

[18] A. Al-Khazraji, N. Essounbouli, A. Hamzaoui, F. Nollet, and J. Zaytoon, "Type-2 fuzzy sliding mode control without reaching phase for nonlinear system," Engineering Applications of Artificial Intelligence, vol. 24, no. 1, pp. 23-38, 2011.

[19] X. P. Do, K. Shah, and S.-B. Choi, "Damping force tracking control of MR damper system using a new direct adaptive fuzzy controller," Shock and Vibration, vol. 2015, Article ID 947937, 2015.

[20] D. X. Phu, N. V. Quoc, J.-H. Park, and S.-B. Choi, "Design of a novel adaptive fuzzy sliding mode controller and application for vibration control of magnetorheological mount," Proceedings of the Institution of Mechanical Engineers Part C: Journal of Mechanical Engineering Science, vol. 228, no. 13, pp. 2285-2302, 2014.

[21] H.-Y. Chen and S.-J. Huang, "Functional approximation-based adaptive sliding control with fuzzy compensation for an active suspension system," Proceedings of the Institution of Mechanical Engineers, Part D: Journal of Automobile Engineering, vol. 219, no. 11, pp. 1271-1280, 2005.

[22] S. D. Nguyen, W. Kim, J. Park, and S.-B. Choi, "A new fuzzy sliding mode controller for vibration control systems using integrated-structure smart dampers," Smart Materials and Structures, vol. 26, no. 4, Article ID 045038, 2017.

[23] N. Karnik and J. Mendel, "Introduction to type-2 fuzzy logic systems," in Proceedings of the 1998 IEEE International Conference on Fuzzy Systems IEEE World Congress on Computational Intelligence, pp. 915-920, Anchorage, AK, USA. 
[24] A. Khosravi, S. Nahavandi, D. Creighton, and D. Srinivasan, "Interval type-2 fuzzy logic systems for load forecasting: a comparative study," IEEE Transactions on Power Systems, vol. 27, no. 3, pp. 1274-1282, 2012.

[25] N. N. Karnik and J. M. Mendel, "Operations on type-2 fuzzy sets," Fuzzy Sets and Systems. An International Journal in Information Science and Engineering, vol. 122, no. 2, pp. 327-348, 2001.

[26] C.-Y. Yeh, W.-H. R. Jeng, and S.-J. Lee, "An enhanced typereduction algorithm for type-2 fuzzy sets," IEEE Transactions on Fuzzy Systems, vol. 19, no. 2, pp. 227-240, 2011.

[27] R. S. Bijan, S. Mohammad, and R. Mehdi, "Control of Active Suspension System: An Interval Type-2 Fuzzy Approach," World Applied Sciences Journal, vol. 12, no. 2, pp. 2218-2228, 2011.

[28] W. Y. Gong and Z. H. Cai, "Differential evolution with rankingbased mutation operators," IEEE Transactions on Cybernetics, vol. 43, no. 6, pp. 2066-2081, 2013.

[29] I. Simon, Handbook of railway vehicle dynamics, CRC Press Taylor and Francis Group, 2006.

[30] V. K. Garg, Dynamics of railway vehicle systems, Academic Press, Harcourt Brace Jovanovich, 1984.

[31] S. D. Nguyen, H. D. Vo, and T. Seo, "Nonlinear adaptive control based on fuzzy sliding mode technique and fuzzy-based compensator," ISA Transactions, vol. 70, pp. 309-321, 2017.

[32] S. D. Nguyen, H.-V. Ho, T.-T. Nguyen, N. T. Truong, and T.-I. Seo, "Novel fuzzy sliding controller for MRD suspensions subjected to uncertainty and disturbance," Engineering Applications of Artificial Intelligence, vol. 61, pp. 65-76, 2017.

[33] X. Liu, M. R. Saat, and C. P. L. Barkan, "Analysis of causes of major train derailment and their effect on accident rates," Transportation Research Record, vol. 2289, pp. 154-163, 2012. 


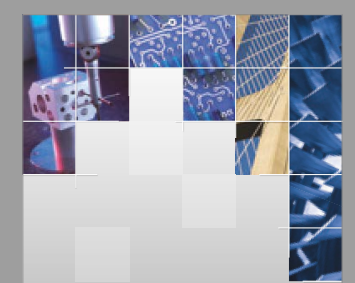

\section{Enfincering}
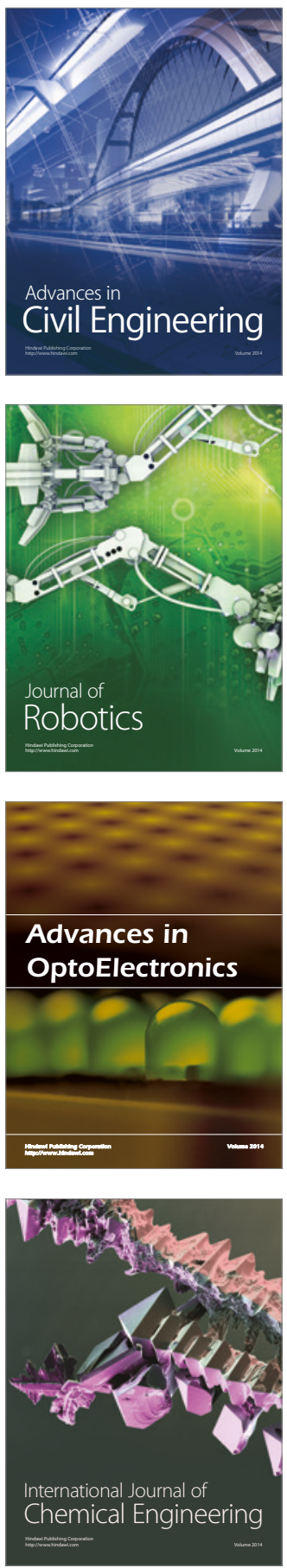

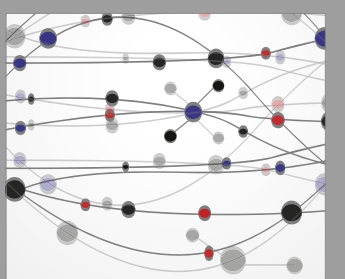

The Scientific World Journal

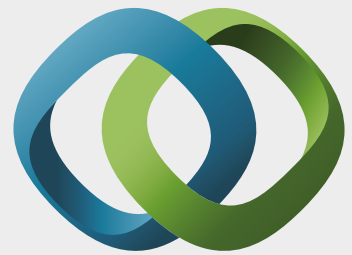

\section{Hindawi}

Submit your manuscripts at

https://www.hindawi.com
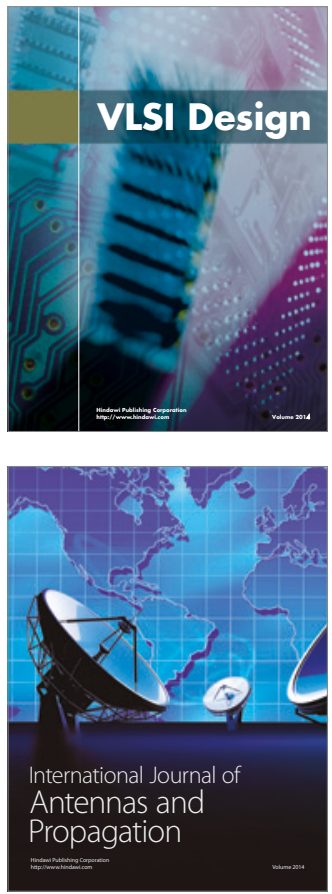

\section{Rotating}

Machinery
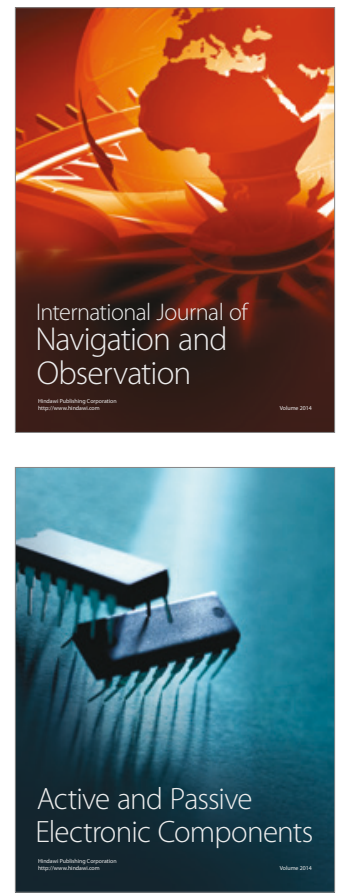
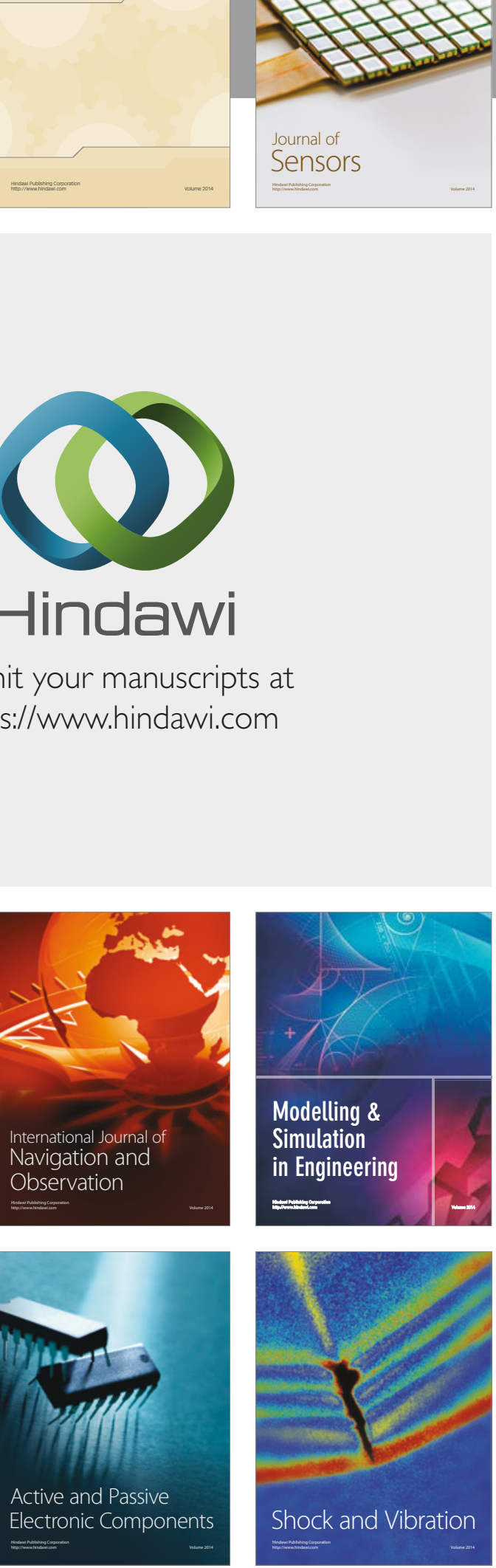
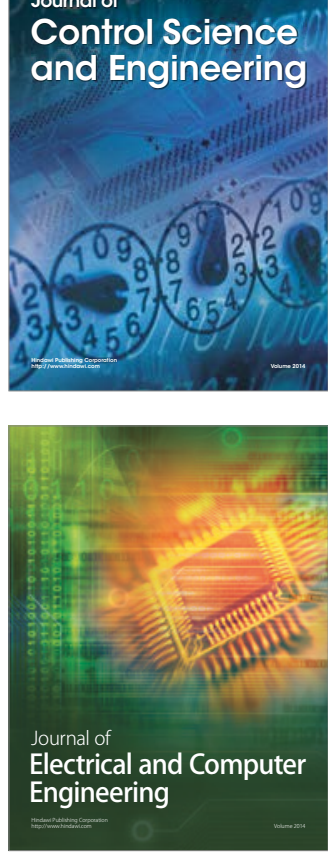

Distributed

Journal of

Control Science

and Engineering
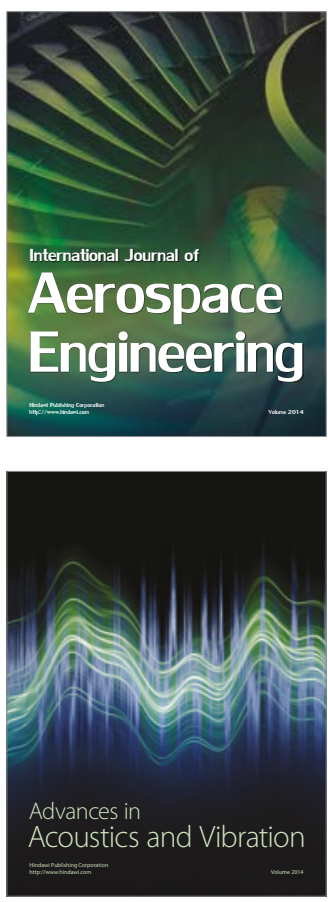

Sensor Networks 